\title{
Fonctionnaires et contractuels en ascension : les conseillers financiers et les recompositions de la mobilité à La Poste
}

Civil servants and contract employees up the professional and social ladder: account managers and changing patterns of mobility in the French Post office

\section{Nadège Vezinat}

\section{OpenEdition \\ Journals}

Édition électronique

URL : http://journals.openedition.org/travailemploi/5459

DOI : 10.4000/travailemploi.5459

ISSN : 1775-416X

Éditeur

DARES - Ministère du Travail

Édition imprimée

Date de publication : 15 novembre 2011

Pagination : 41-54

ISBN : 0224-4365

ISSN : 0224-4365

\section{Référence électronique}

Nadège Vezinat, «Fonctionnaires et contractuels en ascension : les conseillers financiers et les recompositions de la mobilité à La Poste », Travail et Emploi [En ligne], 128 | octobre-décembre 2011, mis en ligne le 12 septembre 2012, consulté le 02 mai 2019. URL : http://journals.openedition.org/ travailemploi/5459; DOI : 10.4000/travailemploi.5459 


\title{
Fonctionnaires et contractuels en ascension : les conseillers financiers et les recompositions de la mobilité à La Poste
}

\author{
Nadège Vezinat $\left.{ }^{*}\right)$
}

La Poste a historiquement été un lieu de promotion sociale, qui a permis aux classes populaires de pénétrer l'univers de la fonction publique au moment où elle était encore une administration d'État. Les transformations organisationnelles récentes qui l'ont affectée l'ont fait sortir de la fonction publique et ont entraîné une déstabilisation du statut d'emploi dominant par l'arrivée massive de contractuels, notamment sur le métier de conseiller financier. Le groupe professionnel des conseillers financiers de la Poste se caractérise par une proportion non négligeable, même parmi les contractuels, de promus internes. Questionner le phénomène de promotion professionnelle au sein de l'entreprise permet dans cet article d'identifier les recompositions qui relèvent du statut d'emploi d'un métier à part au sein de l'organisation mais aussi des mobilités sociales attachées aux trajectoires de chacun.

Tout en contribuant à la reproduction statutaire des élites (DARBel, SchnAPPer, 1969), la fonction publique a aussi joué depuis la fin du XIXe siècle un rôle de promotion sociale pour les diplômés d'origine populaire et rurale (BrowaeYs, Châtelain, 1984), et notamment les femmes (Gollac, 2005). Ce rôle de promotion sociale a été mis en exergue par les monographies consacrées à l'Éducation nationale et aux enseignants du primaire et du secondaire, mais aussi par les travaux sur la Poste, administration de main-d'œuvre par excellence qui, confrontée à un recrutement difficile durant les Trente Glorieuses, s'est alors largement ouverte aux catégories populaires peu diplômées auxquelles elle a permis aussi bien des promotions vers les emplois d'encadrement différenciées en fonction des périodes ainsi que le maintien de possibilités de choix entre des carrières ascensionnelles ou horizontales (JoIN-LAMBERT, 2001 et 2005), que des carrières horizontales sources néanmoins de stabilité et d'honorabilité (CARTIER, 2003). L'appréciation du rôle de promotion sociale d'une administration publique dépend du grade étudié, de la période considérée et des perspectives démographiques, tant les conditions d'accès à la fonction publique varient en fonction de la conjoncture de l'emploi dans le secteur privé (Darbel, SchnAPPER, 1969). Elle dépend aussi bien entendu des critères et des outils de mesure de la mobilité professionnelle et sociale que l'on se donne. Qu'est-il advenu de ce rôle traditionnel de promotion sociale de la Poste, dans le contexte des années 1990 et 2000, marqué par des réformes menant lentement vers la privatisation et par des évolutions de l'emploi et des réorganisations de la gestion du personnel?

Pour répondre à cette question, nous proposons de centrer notre étude sur le métier de conseiller financier, dans la mesure où il a permis aux contractuels - des agents soumis au Code du travail et ne bénéficiant pas du statut de fonctionnaire - d'entrer massivement à la Poste et où il leur a donné l'opportunité d'évoluer du grade d'agents d'exécution à cadres après la réforme de 1991 consacrant la transformation de l'administration d'État en entreprise publique. Ce métier est le produit de transformations organisationnelles qui ont agrégé différents sous-groupes chargés de l'activité financière depuis les années 1970. Il s'est développé récemment avec le recrutement de diplômés de formations de type BTS, notamment des femmes. Dans quelle mesure ce métier de conseiller financier a-t-il effectivement été le support de parcours de mobilité professionnelle ascendante et d'ascension sociale ? Et quel est, dans ces processus, le rôle joué par le statut d'emploi (contractuel ou fonctionnaire)? L'insertion de la Poste dans le marché concurrentiel a entrainé un changement de statut de l'entreprise publique en 1991 et a modifié l'activité de travail des agents, en favorisant notamment le développement des services financiers jusqu'à la création en 2006 de la Banque postale. La Poste a dû pourvoir en ressources humaines sa branche financière et, pour fidéliser les recrues, leur créer des filières de mobilités. Ce métier de conseiller financier constitue ainsi un observatoire singulier mais significatif de l'évolution des phénomènes de mobilités dans la Poste des années 1990 et 2000. 


\section{Encadré 1 \\ Méthodologie}

Trois sources quantitatives sont utilisées dans cet article : une enquête syndicale réalisée en 20052006, une enquête de recherche ad hoc ayant eu lieu en 2007, toutes deux conduites par questionnaire, et une base de données d'entreprise obtenue en 2008. Ces trois sources présentent chacune des avantages et des inconvénients dans leurs apports respectifs.

Si la base d'entreprise ( $n=9$ 987) est exhaustive, elle ne comporte que peu d'informations (statut d'emploi, genre, grade, lieu d'exercice de l'activité). Le questionnaire syndical est plus restreint $(n=610)$ et comporte des biais liés à la fois à son origine et à son mode de passation. II va toutefois plus loin dans l'interrogation des mobilités professionnelles car il intègre des questions sur les salaires, les possibilités de promotion, les carrières, les conditions d'exercice du travail, etc. Le questionnaire ad hoc conçu dans le cadre d'une thèse $(n=166)$ a permis de récolter en deux temps des réponses à plus de 200 items : une phase de passation par courrier en province s'est soldée par 128 retours sur les 254 envois initiaux (soit un taux de retour de plus de $50 \%$ ) et une phase de passation à Paris lors d'une rencontre de conseillers financiers a permis d'obtenir trentehuit retours supplémentaires. Au regard du faible nombre de réponses, ce matériau peut en pratique être considéré comme «qualitatif». Son intérêt est de permettre, au moins sous forme d'hypothèse, d'aborder la question de la mobilité sociale grâce à la reconstitution possible des parcours biographiques et professionnels de chacun des répondants.

Pour compléter l'apport de ces questionnaires, une enquête par entretiens semi-directifs a été réalisée auprès de trente-quatre conseillers financiers en exercice entre 2006 et 2008 et dixhuit autres agents de la Poste : cinq membres de la direction départementale, cinq de la direction nationale, trois receveurs retraités, cinq directeurs d'établissement ayant été (ou non) conseillers financiers. La combinaison de ces méthodes a permis de saisir des données objectives et subjectives en vue de les croiser les unes avec les autres. Les caractéristiques individuelles et étapes de la carrière (diplôme, statut matrimonial, métiers ayant précédé celui occupé au moment de l'entretien, affiliation aux syndicats et associations professionnelles, etc.) constituent un ensemble de faits objectifs qui sont mis en perspective avec les éléments de discours plus subjectifs (les représentations que les enquêtés se font de leur métier, la reconnaissance des clients, des collègues, de la hiérarchie, les images associées au métier comme aux perspectives professionnelles possibles, etc.). Le croisement de ces données s'avère utile pour dresser des profils et illustrer des trajectoires typiques et atypiques de conseillers financiers.

\section{L'apparition du métier de conseiller financier à la Poste : des premiers recrutements par le marché interne}

La Poste compte un peu moins de 300000 agents et les conseillers financiers n'en représentent qu'une petite part (10000 personnes environ, soit à peine plus de $3 \%$ des «postiers» en 2008). Pourtant ce groupe professionnel, mis en place officiellement par la création d'une fiche de poste en interne en 1991, s'est imposé progressivement dans le cadre d'une réorganisation de l'entreprise qui a abouti en 2006 à la création de la Banque postale. En effet, malgré sa faiblesse numérique relative, ses effectifs ont décuplé en moins de vingt ans (voir graphique infra) et l'activité des conseillers financiers occupe désormais une part considérable de l'activité de l'ensemble du bureau de poste. La «statistique 539 », outil établissant la mesure de la charge écoulée dans les bureaux de poste (tant en nombre d'opérations qu'en chiffre d'affaires), permet de dénombrer les opérations liées au courrier comme aux services financiers et effectuées par les différents agents du bureau ${ }^{(1)}$. À titre d'exemple, pour l'année 1989, «le poids des services financiers dans l'activité guichet représente, en zone rurale, $60 \%$ de la production guichet (contre 53,5\% pour l'ensemble des bureaux de poste)»(2). Les services financiers occupant une part importante de l'activité des bureaux de poste, les conseillers financiers en tirent un élément de légitimité interne. Ce groupe professionnel a néanmoins été peu investi par les syndicats : il était trop petit au moment de sa mise en place en 1991 pour les intéresser; et les conseillers financiers, qui sont moins syndiqués que d'autres postiers, ne constituent pas une cible privilégiée des organisations professionnelles. L'essor du métier de conseiller financier a coïncidé avec le recrutement massif de contractuels et cette nouvelle population a de fait peu intéressé les syndicats, alors centrés sur la défense des intérêts des fonctionnaires mis en danger par la réforme de 1991.

Le métier de conseiller financier est officialisé en 1991 et leur nombre croît sensiblement à partir de ce moment-là. L'administration d'État étant devenue une entreprise publique, la réforme du statut de l'entreprise a profondément modifié la structure du salariat postal : comme le cadre juridique et la situation des agents ne relèvent plus directement de l'État, l'emploi des contractuels y a été facilité. Il apparaît sous la forme d'un recours autorisé pour pallier les manques en interne : les recrutements ont d'abord eu

(1) Cet instrument de mesure de l'activité existe dans tous les bureaux de poste, que ceux-ci disposent ou pas de conseillers financiers.

(2) SNA LP, 2002/110-24, La Poste, direction générale, CEDEVE (Centre commun de documentation économique et de veille environnementale), «Les services financiers de La Poste», 9 avril 1996, p. 70. 
lieu sur le marché interne avant de puiser dans des marchés externes pour combler les besoins croissants en conseillers financiers (voir graphique infra). Le projet de loi adopté le 11 avril 1990 stipule que : «lorsque les exigences de l'organisation de certains services ou la spécificité de certaines fonctions le justifient, les exploitants publics peuvent employer, sous le régime des conventions collectives, des agents contractuels, dans le cadre des orientations fixées par le contrat de plan» (art. 30).

Dès 1993 un nouveau système, dit de «reclassification», se substitue aux grades de la fonction publique et établit une grille des emplois fondée sur la notion de classe et le «niveau de fonction» ${ }^{(3)}$. Le grade exprime le niveau hiérarchique du fonctionnaire dans son cadre d'emplois (I, II, III, IV), chaque grade est découpé en trois niveaux de fonction $(1,2,3)$. Il y a quatre classes différentes à la Poste, la classe IV correspondant aux cadres supérieurs, la classe III aux cadres. À titre d'exemple et de comparaison, en 1991, un facteur appartient à la classe I tandis que le conseiller financier entre directement dans la classe II. 3. De nombreux guichetiers et assistants commerciaux de grades II. 1 et II. 2, ainsi que des facteurs de grades I. 1 à I. 3 deviennent conseillers financiers entre 1991 et 1993. Il s'agit d'une promotion dans la mesure où la classification obtenue à l'issue de la mobilité professionnelle est supérieure à celle de départ. Ces mobilités peuvent intervenir rapidement ou tardivement dans les carrières de chacun : elles dépendent moins du cycle de vie des agents que du cycle organisationnel au sein duquel elles s'inscrivent. Ces mobilités résultent ainsi souvent du choix du supérieur hiérarchique. Les promus internes ont déjà fait leurs preuves dans d'autres métiers, l'évaluation annuelle du supérieur direct est en général bonne et lui a permis de déceler des qualités commerciales qui pourraient être davantage utilisées. Le recours aux contractuels a été rendu possible à partir de 1991 mais en 1996, ceux-ci ne représentent que 19,4\% des conseillers financiers (Ріотет, 2002, p. 132), ce qui signifie que le marché privilégié pour les conseillers financiers reste dans un premier temps le marché interne d'où proviennent la plupart des recrutements.

Après s'être fait remarquer par son supérieur, cet agent a ainsi été affecté plus spécifiquement sur le commercial :

«[...] il y avait un collègue de la direction qui était détaché pour le commercial, qui est venu me voir et qui m'a demandé si je serais intéressé par le commercial, et en 1981 j'ai commencé à faire de la CNP [Caisse nationale de prévoyance], et des contrats prévoyance.
Entre-temps j'avais acheté un appartement à [ville de province], et je faisais des fiches de voux pour cette ville, et j'ai été nommé à [ville de province] le ler décembre 1982. Et j'ai fait une journée au guichet, et le chef d'établissement est venu me voir, et il m'a dit: "J'ai vu ta formation, je souhaite ouvrir un cabinet financier" et le 2 décembre 1982 j'ai commencé le cabinet financier.

—Et ça se passait comment? c'était un bureau?

- Non, c'était un petit coin au guichet, et puis on faisait autre chose, le matin je triais les boîtes postales, le soir je faisais le courrier, mais entre-temps si un client était intéressé par un renseignement ou un placement, je m'occupais de lui.

- Vous étiez le spécialiste de ces questions-là dans le bureau?

- Voilà! Alors je me suis formé sur le tas, et par des formations aussi, et je crois que c'est en 1985 que la Poste a créé le métier de COPOFI (conseiller postal et financier), et j'ai commencé à visiter les entreprises pour les envois en nombre, les machines à affranchir, les dossiers de société. Sur le financier j'ai eu quelques formations sur la Bourse, les obligations, les actions. À l'époque il y avait un attaché commercial CNP à la direction, qui faisait de la formation CNP. Et puis il y avait des challenges pour gagner un repas, un voyage et celui qui gérait ça, c'était lui. Et en 1991, je me suis entièrement consacré au financier. Il y avait un choix à faire entre aller vers le courrier ou le financier, moi ayant toujours eu des attaches vers la finance, j'ai préféré aller vers le financier, surtout que le receveur de l'époque m'avait mis un rapport favorable, et je suis rentré dans le métier de conseiller financier sans passer d'examen. »

(Homme, né en 1950, fonctionnaire, niveau bac, entré à la Poste en 1969, COPOFI en 1985, puis COFI en 1991, il l'est toujours, marié, deux enfants, père ouvrier d'usine et mère au foyer.)

Le fonctionnaire, guichetier ou facteur, qui souhaite devenir conseiller financier doit d'abord convaincre son chef d'établissement de sa motivation à effectuer ce travail. Les mobilités en cours de carrière sont donc liées non à un concours (avec des épreuves écrites et un oral) comme pour les corps anciens (ou métiers reconnus) des receveurs ou du service de la distribution et de l'acheminement, mais à une affectation à l'initiative du supérieur hiérarchique. Il n'y a en effet pas eu d'ouverture de concours de fonctionnaires pour occuper l'emploi de conseiller financier. Ceux qui occupaient déjà une fonction financière étaient directement affectés sur ce poste, les autres passaient un examen composé de différentes épreuves (dont une mise en situation commerciale). 
Une contractualisation "sans précarisation " progressivement orchestrée dans les années 1990

Au cours des années 1990, le recours aux contractuels semble cependant progressivement nécessaire pour deux séries de raisons qui se cumulent. D'abord, les besoins en conseillers financiers augmentent tant à partir de 1995 (voir graphique infra) que les processus de promotion interne ne permettent plus de pourvoir les postes avec des mobilités de fonctionnaires. Ensuite, il y a progressivement une pénurie de fonctionnaires candidats : ceux qui avaient l'envie, la capacité et la possibilité d'obtenir cette mobilité l'ont saisie dès les premières années (entre 1990 et 1995, un certain nombre d'assistants commerciaux ou de guichetiers initiés aux produits financiers ont postulé pour évoluer en interne sur ces postes), ce qui laisse supposer que ceux qui restaient, à l'exception des nouveaux venus, ne disposaient pas des compétences nécessaires.

Graphique : Évolution du nombre de conseillers financiers de la Poste tous grades confondus entre 1990 et 2008

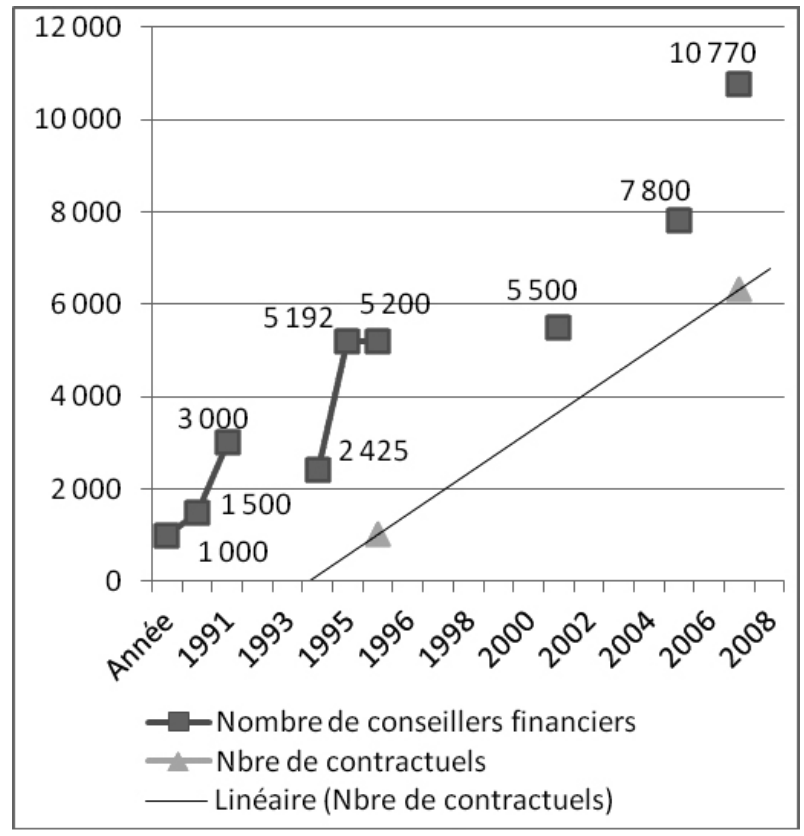

Lecture : En 1991, les conseillers financiers de la Poste sont 1 500. Ils passent au nombre de 3000 en 1992.

Sources : Références, n 40, 1992, p. 9; Sénat, Réponse du ministère des Postes à une question écrite, JO Sénat, 19 décembre 1991, p. 2866 ; Références, no 40, 1992 p. 8; Sénat, Réponse du ministère des Postes à une question écrite, JO Sénat, 19 décembre 1991, p. 2867; F. PioteT, 2002, p. 132; Service national des archives de La Poste (SNA LP) 2000/80-20; Banques des particuliers, avril 1996; Références, no 57, juillet-août 1995, p. 26; Forum, mars 2008.
Le nombre de conseillers financiers double au cours de l'année 1995 (entre 1995 et 1996, on passe de 2425 conseillers financiers à $\left.5537^{(4)}\right)$ et conduit à un recrutement plus intensif de contractuels. La proportion des contractuels et fonctionnaires suit alors des tendances opposées au sein de l'entreprise postale : les derniers recrutements de fonctionnaires, et des concours de facteurs ont lieu en 2000 (DE LA Burgade, Roblain, 2006, p. 80); ils sont ensuite remplacés par des recrutements de contractuels ciblés par métier. En 2002, la Poste compte 323375 agents, dont 227761 fonctionnaires et 108621 salariés de droit privé(5).

Contrairement à l'histoire du métier de trieur pour lequel le recours ancien aux non-titulaires a participé d'une précarisation de l'emploi postal (MAHOUCHE, 2007), la logique qui a présidé à l'embauche de salariés de droit privé sur les postes de conseillers financiers relève d'une logique de professionnalisation. Le recours aux contractuels dans les services financiers ne suit pas en effet les mêmes logiques que dans les centres de tri où les agents contractuels «apportent une certaine flexibilité interne dans le fonctionnement des établissements aux niveaux de l'organisation du temps de travail et de la répartition des ressources sur l'activité» (HARVARD, 2000, p. 92). Les conseillers financiers représentent un métier demandant des compétences spécifiques et sur lequel le recrutement s'avère difficile, après que des vagues successives de promotions internes de fonctionnaires ont assuré son développement entre 1990 et 1995.

Les produits financiers s'affinant, les compétences se sont faites plus pointues et la promotion interne des fonctionnaires a été rejointe - et même dépassée à partir de 1996 - par celle des contractuels embauchés sur des emplois de guichetiers ou de facteurs. Le niveau de qualification des conseillers financiers (voir tableau 1) augmente donc progressivement : depuis 2001, un bac +2 banque ou vente est nécessaire pour être recruté comme conseiller financier.

Le niveau de diplôme des contractuels, supérieur à celui des fonctionnaires, correspond d'abord au relèvement des exigences scolaires qui s'est opéré à l'entrée sur le marché du travail. Entre les cohortes recrutées au début des années 1990 et celles recrutées après 1995, des effets d'âge ont encore accru les différences entre les générations, celle des promus internes moins diplômés (plus âgés) et celle

(4) Piotet Françoise, Correia Mario, Lattès Claude, LÉvy Catherine, PIAUD Florence, Les conseillers financiers de la Poste. Synthèse des résultats de la recherche conduite en 1996 et 1997, Mission Recherche La Poste.

(5) Fonds privé Nadège Vezinat, La Poste, direction prévention, santé et sécurité au travail, «Les risques professionnels des postiers », Dr Claude Miguet (DPSST), Valérie Bougault (Mission Recherche), Dr Brigitte BANCEL CABIAC (DPSST), mai 2005, p. 2. 
des recrutés externes plus qualifiés (plus jeunes). Le profil recherché s'est peu à peu transformé en même temps que le métier. Le type de diplôme (des BTS validant des compétences commerciales) recherché dans le cadre de ces recrutements extérieurs est également significatif de l'importance donnée aux qualités de vendeur des conseillers financiers, ces dernières étant davantage valorisées (ou autant en tout cas) que des compétences techniques financières.

Tableau 1 : Niveaux de diplômes en fonction des statuts en 2005-2006

\begin{tabular}{|l|c|c|c|}
\hline $\begin{array}{c}\text { Statut/niveau } \\
\text { de diplôme }\end{array}$ & Contractuel & Fonctionnaire & Total \\
\hline Inférieur au bac & $3 \%$ & $\mathbf{2 4} \%$ & $10 \%$ \\
\hline Bac & $7 \%$ & $\mathbf{4 5} \%$ & $19 \%$ \\
\hline Bac $+\mathbf{1}$ ou $+\mathbf{2}$ & $\mathbf{6 4} \%$ & $27 \%$ & $52 \%$ \\
\hline Supérieur à bac $+\mathbf{2}$ & $\mathbf{2 6 \%}$ & $4 \%$ & $19 \%$ \\
\hline Effectifs & 413 & 194 & 607 \\
\hline
\end{tabular}

Lecture : $24 \%$ des conseillers financiers fonctionnaires ayant répondu à l'enquête ont un diplôme inférieur au bac.

Champ : conseillers financiers, clientèle en patrimoine, «Les conditions de travail des commerciaux du réseau grand public de La Poste».

Source : résultats CFDT-F3C, Enquête TEQ $(n=610)$.

Le graphique reproduit plus haut fait apparaître trois étapes dans l'évolution du nombre de conseillers financiers entre 1990 et 2008. Le premier moment fort a lieu en 1992 : le groupe des conseillers financiers connaît alors un appel d'air; il se compose de 3000 agents, encore essentiellement des fonctionnaires. Pour expliquer la légère diminution du nombre de conseillers financiers entre 1992 et 1995, plusieurs hypothèses peuvent être formulées : il y a d'abord celle d'un tarissement au début des années 1990 du recours aux fonctionnaires (les plus aptes à devenir conseillers financiers le sont devenus), puis celle liée à la création de filières de promotion qui permettent à un certain nombre de conseillers financiers d'évoluer vers d'autres emplois postaux entre 1993 et 1995. La création de voies de mobilité a contribué à construire la filière du métier de conseiller financier puisque des parcours professionnels au sein de la poste financière sont permis grâce à des évolutions de carrière. S'il donne satisfaction, le conseiller financier peut rester en poste ou évoluer sur des emplois d'expert financier ou de manager. La progression de carrière d'un fonctionnaire (une grande majorité des postiers en 1990 et encore $37 \%{ }^{(6)}$ de la filière financière en septembre 2008) dépend des règles de la fonction publique et se traduit par le relèvement de l'indice et le changement de grade : la Poste a donc dû créer un autre grade pour donner au conseiller financier un débouché. Depuis 1993, il peut devenir «chargé

(6) Pourcentage calculé à partir de la base de données exhaustive fournie par l'entreprise en 2008. de clientèle» (rebaptisé «conseiller clientèle»). Un troisième grade a été mis en place pour la ligne financière en 1995, celui de conseiller spécialisé en patrimoine ou en immobilier.

Le recours aux contractuels étant encore limité avant 1995, les hypothèses avancées ci-dessus viennent expliquer l'évolution de la courbe des conseillers financiers. En 1995, le groupe double de volume, passant, en moins d'un an, de 2425 à plus de 5000 conseillers financiers. Le troisième temps fort a lieu en 2006, au moment de la création de la Banque postale, et se traduit par une nouvelle augmentation du nombre de conseillers financiers. Des campagnes ponctuelles de recrutement (en 1991, 1995, 2002 et 2007), dont les effets sont visibles sur la courbe, indiquent que l'affermissement et la montée en puissance de ce groupe professionnel n'ont pas été linéaires.

$\mathrm{Si}$, en 1996, les contractuels ne représentent que 19,4\% des conseillers financiers (Ріотет, 2002, p. 132), d'après l'enquête CFDT, la proportion fonctionnaires/contractuels s'est inversée en 2005 : la proportion des contractuels atteint désormais $68 \%$. La proportion entre contractuels et fonctionnaires se serait ainsi renversée en moins de dix ans sans pour autant que le métier de conseiller financier semble marqué par une insécurité professionnelle. Les conseillers financiers se situent en effet dans une situation d'emploi «stable» car ils sont tous soit fonctionnaires, soit en CDI. Cette sécurité relative des situations professionnelles vise à limiter le turn-over, c'est-à-dire les départs des conseillers financiers de leur poste. Le fait que l'entreprise cherche par ailleurs à réduire le taux de turn-over des conseillers financiers en CDI indique que la stabilité de l'emploi ne suffit toutefois pas à éviter que certains quittent leur emploi.

Le taux de turn-over permet de comprendre l'évolution du marché interne postal constitué des différents grades de conseillers financiers. En 1995, $18 \%$ des conseillers financiers n'exercent plus cette fonction un an plus tard. À partir du moment où les fonctionnaires sont moins recrutés sur ce métier, le taux de turn-over baisse progressivement pour eux. Cette diminution s'explique par le recrutement concomitant de contractuels qui entraîne une recomposition des promotions. En 1999, le turn-over des fonctionnaires, en moyenne plus âgés, atteint $10 \%$ tandis que celui des contractuels passe en dessous de $20 \%{ }^{(7)}$. Cette baisse semble démontrer que les conseillers financiers fonctionnaires restent plus longtemps que précédemment dans cette fonction. Elle ne permet cependant pas de savoir si les fonctionnaires partent moins en raison d'une volonté

(7) Piotet Françoise, Correia Mario, Lattès Claude, 2002, Les Conseillers financiers, le métier et son exercice, rapport de recherche pour la Collection de la Mission de la Recherche de La Poste, p. 34. 
de rester conseillers financiers ou d'un désir de promotion inassouvi. L'âge comme les opportunités de carrière ne sont pas les mêmes pour les fonctionnaires et les contractuels et peuvent expliquer en partie les différences observées entre les deux populations.

La réduction du taux de turn-over des conseillers financiers peut également signifier que ce métier est susceptible de constituer une opportunité de carrière qui ne nécessite pas de changement d'employeur et évite ainsi tout risque de chômage (Amossé, 2003). La conjoncture joue un rôle important dans l'appréciation de son emploi : en dehors du tournant du millénaire, le contexte des années 1990 et 2000 est aux emplois raréfiés et difficiles à obtenir, même pour les jeunes diplômés, et le nombre de contrats précaires proposés est en augmentation. Cependant, le développement de marchés de travail concurrentiels a aussi entraîné une revalorisation du métier de conseiller financier. En effet, avec un recrutement passé d'un niveau bac à un diplôme bac +2 (Cossalter et al., 2007) dans un contexte de relèvement des exigences scolaires (CHAUvEL, 1998), le métier de conseiller financier apparaît relativement préservé, les contrats prenant majoritairement la forme de CDI.

Pour les contractuels, le départ vers un établissement bancaire concurrent est rendu possible par les processus de mobilité externe entre établissements bancaires (COSSALTER et al., 2007) qui facilitent les recrutements de conseillers. Un «marché interne» des banques de détail s'est constitué en faisant des mobilités entre réseaux un mode de régulation couramment usité par les banques mais également, dans une certaine mesure, par les conseillers financiers eux-mêmes, expliquant ainsi les démissions rapides de certains d'entre eux après leur formation ${ }^{(8)}$.

\section{Conseiller financier : une oppor- tunité de carrière différente pour fonctionnaires et contractuels}

Si les promotions professionnelles concernent aussi bien les fonctionnaires que les contractuels, elles ne s'opèrent pas selon les mêmes modalités en fonction du statut d'emploi. Dans le cadre de notre enquête, l'analyse des questionnaires a mis au jour que la promotion interne faisait partie des grandes voies d'accès au métier de conseiller financier non seulement pour les fonctionnaires,

(8) Bercot Régine, Bruch Véronique, De Coninck Frédéric, ZARIFIAN Philippe, 2001, «Les relations entre front-office et back-office dans la production des services financiers à la Poste», Collection de la Mission de la Recherche de La Poste, p. 57. mais aussi pour les contractuels, et qu'elle concernait même près des deux tiers des répondants. En effet, au regard de l'enquête ad hoc (qui ne représente certes qu'un nombre limité de conseillers financiers), les contractuels - $63 \%$ de la ligne financière en 2008 - accèdent à ce métier pour $25 \%$ d'entre eux directement à leur entrée sur le marché du travail (après l'obtention d'un diplôme de type BTS le plus souvent), pour $15 \%$ après avoir exercé dans une autre entreprise, et pour $60 \%$ par promotion interne ${ }^{(9)}$. Ainsi coexistent le recrutement externe de jeunes diplômés, d'apprentis, de conseillers expérimentés et, dans une large mesure, celui de contractuels ayant connu une promotion interne.

Depuis la fin des années 1970, la probabilité de devenir cadre en cours de carrière a diminué pour les salariés du secteur privé comme semi-public (BARATON, 2006). Cela ne signifie pas que l'accès à ce statut par une mobilité professionnelle ascendante ait disparu (GADÉA, PoCHIC, 2009). Les conseillers financiers de la Poste en fournissent l'illustration. Et, selon les trajectoires de chacun, le métier de conseiller financier constitue alors un grade de fin de carrière, d'attente de la retraite pour les uns, ou au contraire un tremplin, une voie de passage vers des responsabilités managériales ou financières à la Poste pour les autres. Il permet, notamment pour les jeunes diplômés, d'acquérir ou de développer une employabilité directement transposable dans d'autres banques.

La promotion interne ne touche pas tous les agents de la même façon. Pour les fonctionnaires, ce métier constitue un moyen rapide et efficace de changer d'indice et d'échelon, c'est-à-dire de «monter en grade» (voir portrait de Ludovic, encadré 2).

\section{Encadré 2}

\section{Un fonctionnaire promu interne : de facteur à conseiller financier}

«Moi je suis un vieux postier, même si je n'ai que quarante-trois ans, je suis entré à la Poste en 1982, et puis j'ai commencé un peu comme tout le monde, j'ai passé le concours de facteur, et j'ai été nommé facteur en banlieue parisienne. " Ce postier a vécu toutes les grandes transformations statutaires de la Poste. II les connaît d'autant mieux que ses parents sont fonctionnaires de France Télécom. Après avoir été recruté comme fonctionnaire et envoyé à Paris comme facteur, il demande un rapprochement géographique qu'il obtient en 1992 puis revient en

(9) Ce pourcentage, qui peut paraître très important par rapport aux autres, doit être relativisé puisque la majorité des répondants à l'enquête exerce son activité de conseiller financier en province, où la part de promus internes est sans doute plus forte qu'en région parisienne. Il montre toutefois que ces situations sont loin d'être négligeables. 
province, toujours en tant que facteur. II divorce en 1995, son épouse étant restée à Paris. En 19951996, il devient facteur de secteur, c'est-à-dire qu'il prend la responsabilité d'un groupe de facteurs : « $E t$ donc quand tous les facteurs étaient là, c'était bien, je faisais de la paperasse, mais dès qu'un facteur était malade, absent ou en congé, je le remplaçais. Donc je tournais sur six ou sept tournées. Ça avait son charme car on ne faisait pas toujours la même chose et ça me correspondait parce qu'avoir toujours les mêmes horaires, rencontrer les mêmes gens, sur les mêmes trottoirs, ça va un moment, mais après on se lasse un peu. Donc là, ça m'allait très bien. Et puis comme j'avais plus de temps libre, je me suis consacré à la vente de produits courrier, et c'est mon chef d'établissement qui m'a conseillé de me lancer sur un métier vraiment spécifique à la vente. "

Au départ remarqué pour avoir vendu des produits courrier, il choisit finalement de se positionner sur un concours de COFI (conseiller financier) et non pas de COCO (conseiller courrier). II explique d'ailleurs son choix ainsi : "Donc j'ai réfléchi et COCO, finalement, c'était bouché, on ne pouvait pas aller très loin en tant que conseiller courrier, car à part vendre des emballages et des enveloppes, il n'y avait pas autre chose à faire. Alors que COFI, ça ouvrait à un panel de choses à vendre un peu plus important. " II réussit le concours et devient conseiller financier en 1999. II passe très vite le concours de conseiller clientèle en 2000 et l'était encore au moment de l'entretien en 2007.

Ludovic a préféré la voie financière parce qu'elle lui paraissait offrir davantage d'opportunités de carrière et lui permettre, en tant que fonctionnaire, de connaître un avancement plus rapide. L'idée selon laquelle la Poste, en changeant de statut, verrait remis en cause son rôle traditionnel de promotion sociale des diplômés d'origine populaire doit ici être nuancée. La promotion interne n'a pas disparu mais, concentrée sur les activités financières et commerciales, elle concerne à présent non plus seulement les fonctionnaires, mais également les contractuels, et passe surtout par de nouvelles modalités.

Comparés aux fonctionnaires rémunérés à l'ancienneté, les postiers contractuels, fussent-ils concernés par la promotion, n'en restent pas moins désavantagés (BoufFARTIGUE, 2010; ZARIFIAN, 2003) : leurs rémunérations sont moins élevées et les contrats de travail sont, au moins pour un certain nombre, des contrats courts, à durée déterminée, renouvelés régulièrement. Ces situations ont déjà conduit l'entreprise à être condamnée en de multiples occasions : ainsi, le conseil des prud'hommes de Bordeaux a été saisi en 2009 pour traiter du cas d'une salariée ayant signé 250 CDD et travaillé vingt-trois ans pour la Poste; celle-ci a été condamnée en 2011 à requalifier le contrat de travail et verser des dommages et intérêts. Le métier de conseiller financier présente cependant une configuration singulière par rapport aux autres métiers de la Poste. Alors que la prédominance du CDI y dote les contractuels d'une sécurité d'emploi proche de celle garantie par le statut de fonctionnaire, la différence statutaire passe par les possibilités d'évolution en interne. Et celles-ci avantagent non pas les fonctionnaires, mais les contractuels (VEZINAT, 2009), en leur permettant de franchir plusieurs niveaux ou grades en une seule promotion (ce qui est impossible aux fonctionnaires en raison précisément des obligations liées à leur statut). La Poste applique ce faisant le mode de fonctionnement de certaines banques (Crédit Lyonnais, Société générale par exemple), qui privilégient la promotion interne (COSSALTER et al., 2007, p. 99) en développant des perspectives de carrière pour leur personnel, mettant ainsi un frein au recrutement de jeunes diplômés. En donnant plus facilement et/ou rapidement des possibilités d'évolution à ses agents contractuels, la Poste compense la rémunération postale, légèrement plus faible que celle qui se pratique dans les banques du secteur privé $^{(10)}$ :

«Le métier de conseiller financier, par rapport à ses concurrents, il y a deux notions, il y a une notion de rémunération. On a souvent l'impression qu'on est un peu moins bien payé, une impression en réalité je ne sais pas, mais par contre on a une évolution qui est bien plus rapide. J'ai des amis, qui sont issus d'une même formation que moi, qui ont pris la voie de la banque, ils ne sont pas directeur d'établissement ou directeur de banque au bout de huit ans, ils n'en sont pas encore là. Donc, à la Poste, il y a une capacité à nous aspirer vers le haut, qui est bien plus importante.»

(Homme, né en 1965, contractuel, BTS commercial, entré à la Poste en 1997 comme conseiller financier, conseiller spécialisé en patrimoine en 2000, directeur d'établissement en 2004, marié, un enfant, père employé, mère secrétaire.)

Cet homme considère donc que l'entreprise postale lui offre davantage d'opportunités qu'ailleurs et c'est la raison pour laquelle il venait, au moment de l'entretien, de faire embaucher sa femme à la Poste. Il s'agit pour lui de la faire entrer dans l'entreprise afin qu'elle puisse ensuite, à son exemple, y

(10) En juin 2007, pour réduire la différence entre le niveau de rémunération des conseillers financiers de la Poste et les autres conseillers bancaires, la Poste a choisi de modifier les règles de commissionnement en vigueur jusque-là pour aboutir à une nouvelle répartition des rémunérations des conseillers financiers. La rémunération fixe a été «sensiblement augmentée par le transfert (sous forme d'une prime de fonction commerciale bancaire), à compter du 1 er janvier 2007, d'une partie du commissionnement actuel, correspondant au simple exercice de la fonction de conseiller» (Intranet La Poste). Cette nouvelle politique de rémunération a pour objectif de : «séduire et attirer de nouveaux collaborateurs mais aussi et surtout fidéliser les forces de ventes actuelles, qui font très souvent l'objet de sollicitations de la part des concurrents de la Banque Postale. Le discours de ces derniers est simple : il s'appuie sur un réel déséquilibre de la rémunération des équipes commerciales aux dépens de la rémunération fixe et sur un mode de commissionnement ne reconnaissant pas suffisamment la performance» (Forum, l'information, no 214, janvier 2007, p. 12). 
évoluer, preuve que dans son esprit, les possibilités de promotion interne demeurent largement ouvertes aux agents contractuels.

Le cas des conseillers financiers offre ainsi un intéressant contrepoint aux discours et aux représentations qui tendent à confondre «contractuels» et «précaires». Les conseillers financiers contractuels ne sont, d'une part, pas précaires et, d'autre part, peuvent même connaître des phénomènes de promotion interne. Ils ont fait des études supérieures et peuvent espérer évoluer au sein de leur entreprise et parfois, connaître des parcours de promotion impliquant un accès au statut de cadre. Des marchés internes de ce type ont déjà été identifiés dans d'autres entreprises comme la SECMA (Cousin, 2008) ou la SNCF (Boussard, 2011). Au sein de la Poste, ces occasions d'ascension, bien réelles, restent toutefois rares (rapporté à l'ensemble des contractuels, le nombre des conseillers financiers non fonctionnaires demeure faible(11) ) et ne concernent ainsi qu'une minorité de contractuels, ceux qui parviennent à franchir les barrières érigées par le marché interne.

Les mobilités professionnelles ne passent en effet pas par des procédures identiques selon les statuts d'emploi : alors que le statut de fonctionnaire donne des possibilités formelles de mobilité ascensionnelle à travers différents concours internes, les promotions des contractuels sont marquées par une plus grande individualisation des carrières et une moins grande transparence des éléments qui concourent à l'avancement. Le contraste entre les promotions des contractuels et des fonctionnaires n'est pas propre à la Poste : en effet, la gestion locale et personnalisée des ressources humaines favorise aujourd'hui, dans nombre de grandes entreprises, une individualisation des promotions qui, selon les cas, bloque des carrières (HATZFELD, 2004 pour Peugeot; Boussard, 2011 pour la SNCF) ou crée des opportunités (Guillaume, 2011 pour France Télécom). L'ascension professionnelle qui, à partir des années 1990, repose davantage que par le passé sur les compétences et la bonne volonté commerciale (qu'elles soient certifiées par des diplômes type BTS, des expériences professionnelles dans les grandes entreprises du secteur privé, ou encore par des records de vente de produits postaux) ne revêt donc pas les mêmes formes selon le statut d'emploi.

Reposant sur des mobilités numériquement rares et plus individualisées, les promotions internes de facteurs ou guichetiers qui deviennent conseillers financiers constituent aujourd'hui des «destins improbables» (FERRAND, IMBERT, MARRY, 1995) par rapport à la majorité des postiers d'exécution qui vivent des trajectoires «immobiles». Les chances de promotion du salariat d'exécution du secteur

(11) En 2008, le nombre de conseillers financiers contractuels était établi par la direction à 6317 . public sont en effet aujourd'hui plus limitées du fait de l'évolution du recrutement des cadres, de moins en moins issus de la promotion interne (CARTIER, Retière, Siblot, 2010). Les générations anciennes qui affrontent la concurrence des générations plus récentes, mieux diplômées, voient leurs chances de promotion s'amenuiser au fur et à mesure que les plus jeunes se positionnent sur le métier de conseiller financier et saisissent les possibilités d'évolution. Faire partie des «élus » dans ce contexte de concurrence entre des générations inégalement scolarisées amplifie alors le sentiment d'ascension au sein de l'entreprise postale et lui donne les traits d'une réussite individuelle. Par rapport aux formes de promotion plus collectives mais aussi plus modestes des facteurs des Trente Glorieuses (CARTIER, 2003), la Poste en voie de privatisation des années 2000, paraît proposer des mobilités professionnelles plus ascensionnelles, mais aussi plus rares.

La transformation juridique de la Poste ne signifie pas la fin de son rôle traditionnel de promotion, mais plutôt une individualisation et une spécialisation de celle-ci, au profit de certains groupes professionnels et peut-être de certains profils. Afin d'explorer ces profils, nous tentons à présent de prendre en considération la mobilité sociale des conseillers financiers et de faire ressortir la singularité de leurs trajectoires individuelles.

\section{Des conseillers financiers reconnus "cadres" en 2001 : des parcours d'ascension sociale?}

La mobilité professionnelle, intragénérationnelle, vécue par les conseillers financiers, ne coïncide pas forcément avec leur mobilité sociale, intergénérationnelle, qui relie leur position à celle de leur père ou mère. La promotion interne peut de fait être vécue différemment selon l'origine sociale et la trajectoire familiale : «les personnes [ont] d'autant moins tendance à se juger en mobilité ascendante, à caractéristiques identiques, que leur père occupait une position élevée» (Duru-Bellat, KiefFer, 2006, p. 459).

Si la perception de la mobilité est liée aux origines sociales, elle dépend également des capacités des agents à franchir les épreuves de sélection, à tirer parti de l'individualisation des carrières, à faire reconnaître leur mérite individuel. Le fait d'évoluer plus vite que son collègue ou d'occuper une position sociale davantage considérée que celle de ses parents accentue le sentiment de mobilité. Ainsi, l'octroi en 2001 du statut de cadre pour tous les conseillers financiers a permis d'augmenter ce sentiment de mobilité ascensionnelle. La valorisation du statut de conseiller financier par la Poste a en effet pu limiter la différenciation au sein du groupe : l'emploi à statut qu'occupe le fonctionnaire s'avère 
moins éloigné et sujet à regrets pour l'agent contractuel cadre en CDI.

Ce statut de cadre, accordé indépendamment de leur niveau de diplôme, à tous ceux qui exercent le métier de conseiller financier, n'efface toutefois pas la diversité des parcours antérieurs. Parmi les répondants à l'enquête de la CFDT, $25 \%$ des fonctionnaires possèdent un diplôme inférieur au bac et un peu moins de $50 \%$ ont le niveau bac, alors que $90 \%$ des contractuels ont un niveau supérieur au $\mathrm{bac}(\mathrm{bac}+1 \mathrm{ou}+2$ pour la grande majorité, voire supérieur à bac +2 pour un quart d'entre eux). Le niveau de qualification plus élevé des contractuels favorise leur promotion interne par rapport aux fonctionnaires, de moins en moins nombreux (voir portrait de Sophie, encadré 3) et moins bien dotés en termes de qualifications.

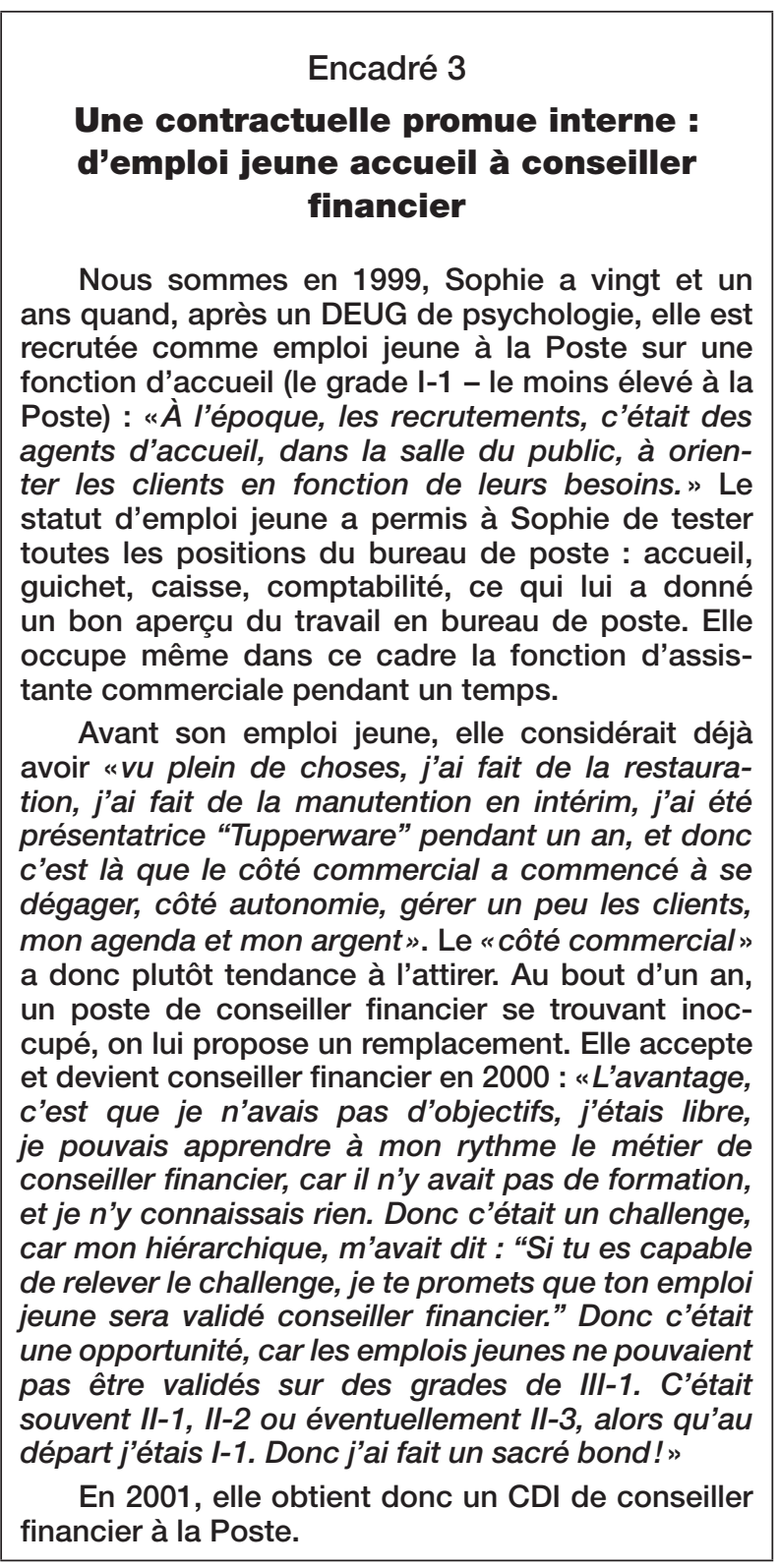

Ce parcours donne à voir une promotion interne rapide et suggère qu'il était encore possible au début des années 2000 d'être contractuelle et de connaître des évolutions de carrière importantes. Pour Sophie, dont le père est employé et la mère au foyer, il s'agit d'une trajectoire ascensionnelle. Elle la considère d'autant plus comme telle que la perception de sa mobilité sociale est sexuée : le décalage plus important pour les femmes (entre une mère peu intégrée au monde professionnel et elle, une femme active) nourrit son fort sentiment de mobilité ascendante. On retrouve ici un constat relevé par DuRu-Bellat et KIEFFER, qui indiquent que les femmes ont «tendance à évaluer leur propre mobilité par rapport à leur mère, souvent inactive (dans les générations plus anciennes), ce qui les conduit souvent à s'estimer en promotion sociale du seul fait de leur activité»» (2006, p. 470). Par ailleurs, dans une conjoncture socio-économique difficile, où les contrats précaires se multiplient et où les diplômés rencontrent des difficultés d'insertion sur le marché du travail, le fait d'obtenir un CDI dans une grande entreprise susceptible d'offrir des évolutions de carrière amène le conseiller financier à percevoir positivement son parcours qui contraste avec les «petits boulots» et les emplois précaires.

Le groupe des conseillers financiers est, comme on l'a vu, un groupe social relativement épargné par le tarissement des emplois de la fonction publique et la précarisation du marché du travail (ils sont tous fonctionnaires ou en CDI). Ce statut professionnel tranche donc avec la détérioration des conditions globales d'insertion des jeunes sur les marchés de l'emploi (LALlement, 2010). Alors que le statut d'emploi des postiers est passé de public à privé - ce qui peut être considéré comme une dégradation en termes de sécurité matérielle -, les jeunes conseillers financiers se trouvent finalement dans une situation plutôt favorable en termes d'emploi par rapport aux autres jeunes : leur métier bénéficie d'une forte demande dans le secteur bancaire et il est peu touché par le chômage. Il présente en outre des perspectives de carrière. Au regard des difficultés des jeunes diplômés à s'insérer sur le marché du travail, les jeunes conseillers financiers sont enclins à se représenter positivement leur position professionnelle : c'est cette représentation que la Poste cherche à renforcer en leur attribuant le statut de cadre.

En effet, si les petits fonctionnaires s'élèvent par le fait d'occuper un emploi à statut, les contractuels, qui les remplacent progressivement, s'élèvent, dans le cas des conseillers financiers, non plus par leur emploi à statut mais par le statut de leur emploi. Le groupe des conseillers financiers s'est en effet vu attribuer en 2001 le statut de «cadre professionnel» (classe III) alors que, jusque-là, il s'agissait d'un métier destiné aux agents de maitrise (de niveau classe II dans l'échelle des emplois postaux). Ce positionnement autour d'un statut de cadre, source de motivation et de promotion sociale, est cependant encore largement déconnecté des attributs traditionnels du cadre (salaires, cotisations à une caisse de retraite cadres) à l'exception des RTT et des dépassements d'horaires, ce qui explique sa réception en demi-teinte sur le terrain, et par les syndicats notamment. Les fonctions concernées par le statut de cadre professionnel (chefs 
d'équipe opérationnels, conseillers commerciaux, chefs d'établissement) sont en effet des métiers qui impliquent des horaires extensifs et, comme ce statut a été créé en 2000, année où la loi sur les trente-cinq heures a été rendue effective dans les entreprises de plus de vingt salariés, on peut y voir une stratégie de contournement du droit du travail par l'entreprise qui accorde en interne le statut de cadre aux métiers les plus chronophages afin de les sortir du dispositif d'application stricte des trente-cinq heures ${ }^{(12)}$.

Une évolution récente doit néanmoins être soulignée, qui suggère une revalorisation plus conséquente du métier de conseiller financier. Derrière la «permanence des positions», il peut y avoir un "changement des conditions" (COUTROT, 2002) : les contractuels de droit privé recrutés depuis le 1 er janvier 2011 ne sont plus en effet affiliés à l'Ircantec mais à l'Agirc-Arrco. En cotisant à une caisse de retraite de cadres, les conseillers financiers de la Poste se différencient donc des conseillers bancaires qui, travaillant sur les mêmes niveaux de fonction, ne sont pas considérés comme des cadres. Ce groupe de postiers, à la frontière du statut cadre, permet finalement de montrer en quoi une requalification de statut sert d'outil de fidélisation d'une main-d'œuvre susceptible de quitter l'entreprise pour la concurrence avec pour conséquence une augmentation du niveau de diplôme des recrutés extérieurs. L'accès à un statut de cadre contribue à repositionner ce métier sur l'échelle des emplois postaux, en l'éloignant des emplois de guichetiers (classe II), pour le rapprocher de ceux des managers de proximité (classe III).

Même si le statut de cadre apparait encore plus symbolique que réel, il revêt une signification en termes de chemin parcouru pour les personnels. Le conseiller financier cité dans l'entretien suivant, tout en minimisant d'abord l'importance de son changement de statut, évoque finalement sa «satisfaction personnelle d'être arrivé jusque-là», c'est-à-dire qu'il a pu devenir cadre, au sein même de l'entreprise où ses parents ont été fonctionnaires de catégorie $\mathrm{C}$ :

«Et le fait de passer cadre, qu'est-ce que ça a changé pour toi?

- Je dirais pas grand-chose. Pas grand-chose parce que je n'ai pas l'impression d'être cadre. Ça a changé un peu mon salaire certes, et puis quand on me demande ma profession, je ne dis pas "cadre", je dis "conseiller financier" ou "conseiller clientèle". Je suis content, je suis cadre, mais ça va pas plus loin.

(12) Le dépassement d'horaires au-delà des trente-cinq heures hebdomadaires est en effet permis pour les cadres qui bénéficient, en théorie, de forfaits jours de RTT pour les compenser; dans la pratique, ces jours de repos supplémentaires ne compensent pas toujours les heures de travail effectuées (BouffartigUE, 2001).
C'est peut-être une satisfaction personnelle d'être arrivé jusque-là, voilà !»

(Homme, né en 1964, fonctionnaire, BEPC, entré à la Poste en 1982, conseiller financier en 1999, divorcé, parents fonctionnaires à France Télécom.)

La mobilité doit certes être appréciée au regard de l'évolution des structures d'emploi (passage de fonctionnaire à contractuel, augmentation du niveau de qualification), mais elle doit l'être également en fonction des trajectoires biographiques individuelles. La pente de la trajectoire professionnelle et la distance avec le milieu social d'origine jouent un rôle dans la perception de la mobilité. Les promus internes sont satisfaits de leur position d'arrivée quand les jeunes diplômés peuvent être déçus de leur parcours. C'est sans doute le cas de la jeune diplômée de vingt-quatre ans, bac +2 , BTS commercial, père fonctionnaire du Trésor public, mère fonctionnaire à France Télécom, tout juste recrutée comme conseiller financier en 2007, qui, quand on lui demande où elle sera selon elle dans cinq ans, répond :

«Moi, dans cinq ans, je me vois assez bien dans un poste de direction, j'aimerais bien DAC (directeur de l'action commerciale) [c'est un poste de cadre supérieur, il y en a environ un par département et ce sont plutôt des agents expérimentés qui l'occupent]... Je crois que comme d'ici un an j'aurais fait le tour du métier [elle vient d'être recrutée au moment de l'entretien], je chercherai à évoluer rapidement en interne.»

Recroisée en 2011, cette enquêtée semble insatisfaite de sa position professionnelle : elle qui se projetait dans un poste de cadre supérieur, alors que cette trajectoire professionnelle n'est concrètement pas réalisable en si peu de temps, est toujours conseiller financier dans le même bureau de poste, c'est-à-dire sans aucune mobilité, même horizontale.

Même si les parcours ascensionnels sont loin d'être majoritaires à l'échelle de l'entreprise, l'ancien modèle de la promotion interne n'a pas disparu. Il perdure dans un contexte organisationnel en évolution profonde, dans lequel le métier même de conseiller financier a tellement changé qu'il ne peut pas être considéré comme une variable fixe à partir de laquelle mesurer la mobilité. Le rapport à la mobilité professionnelle des conseillers financiers dépend toutefois également de la trajectoire parcourue vis-à-vis de ses parents. La mobilité sociale des conseillers financiers est étudiée ci-après à partir d'une enquête par questionnaire réalisée dans le cadre d'une thèse (voir encadré méthodologique en début d'article). Elle s'appuie sur l'étude de données certes de faibles effectifs mais qui sont précieuses en l'absence d'autres sources existantes et ont par ailleurs été enrichies par des entretiens. 


\section{Une diversification des origines sociales des conseillers financiers}

Les origines sociales des conseillers financiers suggèrent que la Poste en voie de privatisation demeure un espace d'ascension sociale pour les diplômés d'origine populaire. Ce type de trajectoires dépend des politiques d'emploi et de gestion des carrières des entreprises plus que des secteurs d'activité : ainsi, dans la grande distribution, alors que Décathlon recrute ses cadres sur son marché interne, Go Sport les embauche à l'extérieur (Dalla Pria, Leroux, 2011). Quand le marché de l'emploi à France Télécom met fin à la promotion interne (Guillaume, 2011), la Poste la maintient sous certaines conditions. Ainsi, cette contractuelle entrée à la Poste en 1991 comme factrice et devenue conseiller financier en 1993, explique-t-elle avoir bénéficié d'une promotion interne rapide qui lui a permis de franchir plusieurs échelons :

«Je voulais être davantage sur du commercial et je commençais à éprouver une sorte de lassitude, un jour j'ai vu un appel à candidature pour être conseiller financier, j'ai dit pourquoi pas devenir cadre, et comme les ACO avaient la possibilité de passer de I-3 (grade de facteur) à III-1 (grade de conseiller financier), j'ai tenté ma chance et ça a fonctionné. »

Le fait de devenir cadre en franchissant plusieurs grades d'un seul coup, par une promotion au rang de conseiller financier, constituait une motivation forte pour cette factrice recrutée en 1991, fille d'un père mécanicien et d'une mère ouvrière. Le vocabulaire de la bifurcation («un jour», «j'ai tenté ma chance») suggère un déplacement social tout autant lié aux circonstances qu'au projet individuel. Diplômée d'un DUT GEA, elle décrit ainsi le «concours» de conseiller financier passé en 1993 (c'est en réalité un simple examen, mais elle en parle comme d'un rite de passage sur le modèle du concours des fonctionnaires) :

«J'ai été reçue par le directeur départemental de l'époque et ses collaborateurs, et quelque temps après j'ai été contactée par le directeur commercial qui m'a dit "Voilà, vous avez fait bonne impression au directeur de la Poste, il existe aujourd'hui des postes de conseillers financiers, allez chercher un dossier, remplissez-le, envoyez-le." Voilà! Donc j'ai monté mon dossier et je l'ai envoyé. Et à l'époque, conseiller financier, c'était un super-examen, avec des tests psychotechniques, et ça durait trois ou quatre heures, et nous étions une soixantaine, la plupart, c'étaient des postiers qui n'avaient pas fait d'examen de ce style-là. Donc moi qui sortais de l'école, je me sentais à l'aise par rapport à eux, et en plus, il fallait choisir entre conseiller financier ou délégué commercial sachant que conseiller financier, c'était la vente aux particuliers et délégué commercial, la vente aux entreprises. Et donc j'ai choisi conseiller financier parce qu'on m'avait dit que c'était plus accessible et plus facile, mais je ne savais pas ce que c'était finalement. Donc c'était assez vaste le milieu bancaire à l'époque, c'était un peu nébuleux. Mais moi, mon objectif c'était de bosser. Et je n'étais pas partie pour faire une carrière à la Poste, je ne raisonnais pas ainsi, je voulais juste gagner ma vie. Donc des tests psychotechniques, puis un jury avec au moins cinq personnes, il y avait un "training" où je devais tenir un rôle, et ensuite un entretien avec un psychologue, qui lui m'avait dit que je ne rentrerais jamais à la Poste. Et j'avais vingt et un ans à l'époque.»

Là encore, on retrouve l'idée d'une mobilité peu planifiée («Pourquoi pas devenir cadre?»), incertaine, doublée toutefois de l'assurance que procurent les ressources scolaires, utiles pour se différencier positivement dans le cadre de cette épreuve de sélection.

En interrogeant le positionnement des enfants par rapport à celui de leurs parents (13), il apparaît que les conseillers financiers sont plutôt enfants d'ouvriers (à $25 \%$ ) et d'agricultrices (à $35 \%$ ). Les conseillers financiers fonctionnaires présentent des origines sociales plus modestes que celles des contractuels (pères ouvriers pour $25 \%$ d'entre eux, agriculteurs pour $16 \%$ et artisans-commerçants pour $16 \%$ et mères majoritairement agricultrices). C'est en partie dû aux limites de l'enquête portant sur des effectifs faibles et à un effet de génération entre les fonctionnaires, en moyenne plus âgés, et les contractuels. Cela atteste en tout cas du rôle joué par la Poste, administration d'État, dans la promotion sociale des classes populaires (CARTIER, 2003). Ainsi cet homme, fonctionnaire, aux parents agriculteurs, a voulu entrer à la Poste quand elle était une administration d'État :

«Je voulais entrer à la Poste parce que je voulais être à la Poste.

—Ah oui? pourquoi? Vos parents étaient postiers?

- Non, du tout, mes parents étaient agriculteurs, mais bon moi, je voulais être postier, ça me plaisait sans que je sache trop pourquoi. Je suis postier et jusqu'au bout je serai postier, et on m'entendra pas critiquer la boutique. Mais par contre je me donne à fond et je veux que ça paye.»

(Homme, né en 1951, fonctionnaire, BEPC, entré à la Poste en 1970, conseiller financier en 1991, est CSI (conseiller spécialisé en immobilier) depuis 1995.)

Il a bénéficié d'un emploi stable et a pu évoluer en interne. Il est devenu cadre en même temps que conseiller spécialisé en immobilier et est prêt à s'investir tant que «ça paye», c'est-à-dire que la promotion s'avère

(13) La limite des tables de mobilité sociale tient dans la vision statique qu'elles donnent des trajectoires. En masquant les bifurcations, les «turning points» (BECKER, 1985, p. 126; StRAUSS, 1992, p. 85) dans les parcours individuels, le milieu d'origine n'est généralement apprécié qu'au regard de la position du père, réduite à sa PCS (profession et catégorie socioprofessionnelle). Dans notre enquête, croisant des matériaux qualitatifs et quantitatifs, la PCS de la mère des conseillers financiers est connue et a permis de reconstituer plus finement leurs cheminements biographiques et professionnels. 
possible. Ce parcours professionnel est particulier en ce sens qu'avant même l'officialisation du métier de conseiller financier en 1991, cet enquêté était affecté sur une fonction financière : guichetier, il est devenu en 1983 «détaché CNP», c'est-à-dire qu'en plus de son travail de guichetier, il se rendait disponible pour vendre des produits de la Caisse nationale de prévoyance (CNP). Ces conseillers financiers avant l'heure n'ont donc eu aucune difficulté à devenir conseillers financiers en 1991, d'autant qu'aucun concours ou examen n'existait alors. Ayant une solide expérience, ce postier a pu faire partie des premiers agents conseillers spécialisés.

Bien que sur la voie de la privatisation, la société anonyme La Poste a continué de permettre à des hommes peu diplômés de gravir les échelons, mais ces derniers ont alors de plus en plus subi la concurrence de contractuels, plus jeunes, plus féminisés, plus diplômés. Les recompositions du groupe professionnel expliquent en partie les différences entre les origines sociales des conseillers financiers fonctionnaires et contractuels. Les contractuels appartenant à une génération postérieure à celle des fonctionnaires, ils sont en moyenne plus jeunes de dix ans. Une diversification est ainsi observable pour les contractuels entre ceux qui ont des origines sociales proches des fonctionnaires issus des classes populaires (pères ouvriers pour $25 \%$ et employés de la fonction publique pour $14 \%$ d'entre eux), et ceux qui sont issus des classes moyennes et supérieures (pères cadres d'entreprise et commerciaux pour $17 \%$ et cadres de la fonction publique pour $13 \%$ d'entre eux : voir tableau 2 ).

Tableau 2 : Origines sociales des pères en fonction du statut des conseillers financiers

\begin{tabular}{|c|c|c|c|}
\hline \multirow{2}{*}{$\begin{array}{l}\text { Profession } \\
\text { du père }\end{array}$} & \multicolumn{3}{|c|}{ Statut du conseiller financier } \\
\hline & $\begin{array}{l}\text { Contrac- } \\
\text { tuel }\end{array}$ & $\begin{array}{l}\text { Fonction- } \\
\text { naire }\end{array}$ & $\begin{array}{l}\text { Total } \\
\text { général }\end{array}$ \\
\hline Agriculteur & $9 \%(9)$ & $16 \%(10)$ & $12 \%(19)$ \\
\hline Artisan, commerçant & $6 \%(6)$ & $16 \%(10)$ & $10 \%(16)$ \\
\hline Chef d'entreprise & $4 \%(4)$ & $3 \%(2)$ & $4 \%(6)$ \\
\hline Profession libérale & $1 \%(1)$ & $2 \%(1)$ & $1 \%(2)$ \\
\hline $\begin{array}{l}\text { Cadre de la fonction } \\
\text { publique (catégorie A) }\end{array}$ & $13 \%(13)$ & $5 \%(3)$ & $10 \%(16)$ \\
\hline $\begin{array}{l}\text { Cadre d'entreprise et } \\
\text { commercial }\end{array}$ & $17 \%(17)$ & $11 \%(7)$ & $15 \%(24)$ \\
\hline $\begin{array}{l}\text { Profession intermédiaire } \\
\text { de la fonction publique } \\
\text { (catégorie B) }\end{array}$ & $6 \%(6)$ & $5 \%(3)$ & $5 \%(9)$ \\
\hline $\begin{array}{l}\text { Profession intermédiaire } \\
\text { d'entreprise }\end{array}$ & $0 \%$ & $2 \%(1)$ & $1 \%(1)$ \\
\hline $\begin{array}{l}\text { Employé de la fonction } \\
\text { publique (catégorie C) }\end{array}$ & $14 \%(14)$ & $11 \%(7)$ & $13 \%(21)$ \\
\hline Employé d'entreprise & $6 \%(6)$ & $0 \%$ & $4 \%(6)$ \\
\hline Ouvrier & $25 \%(26)$ & $26 \%(16)$ & $25 \%(42)$ \\
\hline (vide) & $1 \%(1)$ & $3 \%(2)$ & $2 \%(3)$ \\
\hline Total général & $100 \%(103)$ & $100 \%(62)$ & $100 \%(165)$ \\
\hline
\end{tabular}

Lecture : $16 \%$ des conseillers financiers fonctionnaires ont déclaré que leur père était agriculteur.

Champ : conseillers financiers en exercice en 2007.

Source : tableau réalisé à partir de l'enquête par questionnaire passée en $2007(n=165)$.
La profession des mères (voir tableau 3) des conseillers financiers contractuels (surtout des agricultrices, des ouvrières et des employées de la fonction publique) semble moins faire apparaître la dichotomie remarquée dans les PCS déclarées pour les pères mais rappelle que l'hérédité sociale des fonctionnaires est d'autant plus perceptible lorsqu'on prend en considération, en dehors de la situation du père, celle de la mère (DE Singly, THÉLOt, 1989). Le fait qu'un des parents appartienne déjà à la fonction publique apparaît par ailleurs comme un facteur de transmission du statut bien plus déterminant que le niveau de formation (AUDIER, 2000, p. 130). En effet, pour ce qui est de la reproduction sociale, sur les 165 conseillers à avoir répondu au questionnaire, 58 (soit plus de $35 \%$ des répondants de l'échantillon) ont au moins un membre de leur famille qui travaille ou a travaillé à la Poste.

Tableau 3 : Origines sociales des mères en fonction du statut des conseillers financiers

\begin{tabular}{|c|c|c|c|}
\hline \multirow{2}{*}{$\begin{array}{l}\text { Profession } \\
\text { de la mère }\end{array}$} & \multicolumn{3}{|c|}{ Statut du conseiller financier } \\
\hline & $\begin{array}{c}\text { Contrac- } \\
\text { tuel }\end{array}$ & $\begin{array}{l}\text { Fonction- } \\
\text { naire }\end{array}$ & $\begin{array}{c}\text { Total } \\
\text { général }\end{array}$ \\
\hline Agriculteur & $22 \%(23)$ & $55 \%(34)$ & $35 \%(57)^{*}$ \\
\hline Artisan, commerçant & $7 \%(7)$ & $2 \%(1)$ & $5 \%(8)$ \\
\hline Chef d'entreprise & $3 \%(3)$ & $0 \%$ & $2 \%(3)$ \\
\hline Profession libérale & $3 \%(3)$ & $0 \%$ & $2 \%(3)$ \\
\hline $\begin{array}{l}\text { Cadre de la fonction } \\
\text { publique (catégorie A) }\end{array}$ & $5 \%(5)$ & $2 \%(1)$ & $4 \%(6)$ \\
\hline $\begin{array}{l}\text { Cadre d'entreprise et } \\
\text { commercial }\end{array}$ & $2 \%(2)$ & $2 \%(1)$ & $2 \%(3)$ \\
\hline $\begin{array}{l}\text { Profession } \\
\text { intermédiaire de la } \\
\text { fonction publique } \\
\text { (catégorie B) }\end{array}$ & $9 \%(9)$ & $8 \%(5)$ & $8 \%(14)$ \\
\hline $\begin{array}{l}\text { Profession intermédiaire } \\
\text { administrative } \\
\text { d'entreprise }\end{array}$ & $2 \%(2)$ & $3 \%(2)$ & $2 \%(4)$ \\
\hline $\begin{array}{l}\text { Employé de la fonction } \\
\text { publique (catégorie C) }\end{array}$ & $17 \%(18)$ & $3 \%(2)$ & $12 \%(20)$ \\
\hline Employé d'entreprise & $11 \%(11)$ & $5 \%(3)$ & $8 \%(14)$ \\
\hline Ouvrier & $18 \%(19)$ & $16 \%(10)$ & $18 \%(29)$ \\
\hline (vide) & $1 \%(1)$ & $5 \%(3)$ & $2 \%(4)$ \\
\hline Total général & $100 \%(103)$ & $100 \%(62)$ & $100 \%(165)$ \\
\hline
\end{tabular}

* Le nombre important de mères déclarées agricultrices par les conseillers financiers peut masquer une proportion non négligeable de femmes au foyer. Cette dernière catégorie n'étant pas proposée parmi les items de réponses, les répondants ont pu laisser vide le tableau ou indiquer le métier occupé par leur mère quand elle travaillait (donc avant d'être à la retraite ou au foyer).

Lecture : $55 \%$ des conseillers financiers fonctionnaires ont déclaré que leur mère était agricultrice.

Champ : conseillers financiers en exercice en 2007.

Source : tableau réalisé à partir de l'enquête par questionnaire passée en $2007(n=165)$.

Les contractuels enfants d'ouvriers ont entre vingt et cinquante ans et la moitié d'entre eux sont devenus conseillers financiers par promotion interne (par opportunité ou sur la proposition d'un supérieur hiérarchique). Ces promotions plus individualisées et moins transparentes nécessitent dans tous les cas une entente entre le directeur d'établissement 
et le conseiller, qui reconnaît l'importance d'être apprécié par son hiérarchique tant pour ce qui est de la rémunération que des avancements de carrière potentiels :

\section{«Et les rapports avec le chef d'établissement?}

- Ah! c'est très important, c'est lui qui me note, c'est lui qui fait la prime qualité, qui joue un rôle pour une part de notre rémunération annuelle, et il est là aussi pour notre augmentation, parce que sans bonne notation, les augmentations ne suivent pas, l'évolution de la carrière ne suit pas non plus. »

(Homme, né en 1978, contractuel, BTS en alternance avec la Poste, entré à la Poste en 2001 comme conseiller financier, il l'est toujours, non syndiqué, marié, sans enfant, père facteur.)

Les stratégies des directeurs d'établissement sont néanmoins variables. Le supérieur hiérarchique qui est satisfait de son conseiller financier peut aussi tenter de limiter sa mobilité afin de le garder le plus longtemps possible. C'est ce qu'indique l'enquêté précédent :

«Un DET soit il apprécie son COFI et il va tout faire pour le garder, soit il l'aime pas et il va essayer de le faire évoluer, ça arrive, même si ça va à l'envers de ce qu'il faudrait faire, et puis d'autres qui, quand ça se passe bien, essaient de garder un petit peu le vendeur pour un an ou deux, et puis après ils font le maximum pour que ça se passe bien. Donc il y a des bons managers comme il y a de bons COFI, et quand je dis bons, ce n'est pas au niveau des résultats mais de la mentalité.»

Les parcours professionnels des contractuels ne sont pas non plus linéaires. Une dichotomie apparaît entre les recrutés sur leurs diplômes $(\mathrm{bac}+2$, bac $+3 / 4$ ou bac +5$)$ après 2006 et la création de la Banque postale, et les promus internes (BEPC, BEP/ CAP, bac ou bac +2 ), arrivés plus tôt à la Poste, dans les années 1990, mais qui ne sont devenus conseillers financiers que dans les années 2000, plus proches des fonctionnaires en termes de profils.

Les contractuels promus internes se trouvent en ascension sociale, quand les contractuels, jeunes diplômés, sont recrutés récemment et en début de carrière. Le positionnement par rapport aux parents s'avère d'ailleurs d'autant plus délicat à saisir que les conseillers financiers usent de rhétoriques de justification pour mettre leur sentiment de mobilité sociale en conformité avec leurs parcours. Au final, il apparaît que le clivage des statuts d'emploi en recouvre un autre, peut-être plus décisif pour expliquer les perceptions des promotions, entre deux types de mobilité : celle des promus internes d'origine populaire (fonctionnaires et contractuels) qui conduit au métier de conseiller financier et celle des jeunes diplômés (uniquement contractuels) qui mène vers d'autres grades au sein du groupe des conseillers financiers.

$\begin{array}{ll} & * \\ * & *\end{array}$

L'individualisation des parcours professionnels qui se diffuse dans le monde postal n'exclut pas qu'une mobilité sociale intergénérationnelle ascendante soit constatée, même si elle est moins nette pour les contractuels que pour les fonctionnaires. Les modalités d'ascension au sein d'une organisation sur la voie de la privatisation comme l'est la Poste des années 1990 et 2000, se recomposent. Les perspectives de carrière n'ont pas le même impact selon qu'il s'agit d'un poste de fin de carrière pour un fonctionnaire arrivant en fin de grille indiciaire ou d'un jeune diplômé qui voit ce métier comme un tremplin pour lancer sa carrière professionnelle et valoriser son diplôme. L'emploi de conseiller financier est d'autant plus prisé qu'il est devenu l'enjeu de promotions considérées comme importantes : il correspond à l'entrée dans la classe III, le niveau de grade des cadres. Et il ne concerne qu'un nombre limité d'agents.

Le statut attractif de fonctionnaire a été peu à peu remplacé par celui de cadre, valorisé en interne pour les emplois de contractuels en CDI, notamment ceux situés dans les services financiers. Les salariés y sont à présent moins retenus par un statut d'emploi avantageux que par des perspectives de carrière. Le métier de conseiller financier est en effet le passage obligé pour accéder à des postes à responsabilités (d'expert financier ou de manager). Dans ce cadre, la «mobilité intragénérationnelle [...] vient brouiller le jugement concernant la mobilité intergénérationnelle» (Duru-Bellat, KiefFer, 2006, p. 467). Selon les cas, la trajectoire professionnelle amplifie la distance sociale parcourue ou la fait paraître moins grande. Le fait d'accéder à un statut de cadre amplifie la distance avec des origines sociales modestes et limite celle qui peut exister avec des parents cadres supérieurs.

L'effectivité de la mobilité est toutefois relative : les conseillers financiers sont certes cadres, mais ce statut n'est pas reconnu en dehors de l'entreprise; ils sont diplômés mais dans un monde où le niveau scolaire n'a cessé d'augmenter; ce sont des promus internes évoluant au sein de leur entreprise quand d'autres ne bougent pas, mais la mobilité qu'entraînent ces changements de grades ne concerne qu'un changement de portefeuille de clients, et peut être vue, selon une autre perspective, comme une modification négligeable de leur activité de travail. 


\section{Bibliographie}

Amossé T. (2003), «Interne ou externe, deux visages de la mobilité professionnelle», Insee Première, no 921.

Audier F. (2000), «La transmission du statut dans la fonction publique», Économie et Statistique, no 337-338, pp. 121-133.

BARATON M. (2006), «De la difficulté à devenir cadre par promotion», Insee Première, no 1062.

BeCKer H.S. (1985), Outsiders : études de sociologie de la déviance, Paris, Métailié.

Bouffartigue P. (2001), Cadres : la grande rupture, Paris, La Découverte.

Bouffartigue P. (2008), «Précarités professionnelles et action collective. La forme syndicale à l'épreuve», Travail et Emploi, no 116, pp. 33-43.

Bouffartigue P. (2010), «L'emploi à statut au miroir de ses marges. Précaires à la Poste dans les années 2000 », in Cartier M., Retière J.-N., Siblot Y., Le salariat à statut. Genèses et cultures, Rennes, PUR, pp. 287-299.

Boussard V. (2011), «Des cadres de promotion rétifs au changement? L'exemple de la SNCF», in Bouffartigue P., Gadéa C., Pochic S., Cadres, classes moyennes : vers l'éclatement?, Paris, Armand Colin, pp. 93-103.

Browaeys X., Châtelain P. (1984), Les Frances $d u$ travail, Paris, Presses universitaires de France.

CARTIER M. (2003), Les facteurs et leurs tournées, un service public au quotidien, La Découverte, Paris.

Cartier M., Retière J.-N., Siblot Y. (2010), Le salariat à statut. Genèses et cultures, Rennes, PUR.

Chauvel L. (1998), Le destin des générations, Paris, PUF.

Cossalter C., Ledan P., Moussy J.-P., Musseau J., Plessis A., Omnès C., Gastaldi L. (2007), «La gestion des ressources humaines dans les banques », Entreprises et Histoire, Vol. 48, no 3, pp. 92-108.

Cousin O. (2008), Les Cadres à l'épreuve du travail, Rennes, Presses universitaires de Rennes.

Coutrot L. (2002), «Lescatégories socioprofessionnelles: changement des conditions, permanence des positions?», Sociétés contemporaines, no 45-46, pp. 107-129.

Dalla Pria Y., Leroux N. (2011), «Fidéliser par la promotion : un modèle toujours attractif dans la grande distribution », in Bouffartigue P., Gadéa C., Pochic S., Cadres, classes moyennes : vers l'éclatement?, Paris, Armand Colin, pp. 104-115.

Darbel A., Schnapper D. (1969), Les agents du système administratif, Paris, La Haye, Mouton.

De La Burgade E., Roblain O. (2006), «Bougez avec La Poste» : les coulisses d'une modernisation, Paris, La Dispute.

De Singly F., Thélot C. (1989), Gens du privé, gens du public. La grande différence, Paris, Dunod.
Duru-Bellat M., KiefFer A. (2006), «Les deux faces objective/subjective - de la mobilité sociale», Sociologie du travail, Vol. 48, no 4, pp. 455-473.

Ferrand M., Imbert F., Marry C. (1995), «Normaliennes scientifiques et polytechniciennes : des destins improbables?», Regards sociologiques, no 9-10, pp. 101-108.

Gadéa C., Pochic S. (2009), «Des “disparus” bien présents : les cadres issus de la promotion», Éducation permanente, $\mathrm{n}^{\circ} 178$, pp. 9-24.

Gollac S. (2005), «La fonction publique : une voie de promotion sociale pour les enfants des classes populaires?», Sociétés contemporaines, no 58, Paris, pp. 41-63.

Guillaume C. (2011), «La face cachée du management à France Télécom. Les enjeux de carrière dans une entreprise en restructurations », in Bouffartigue P., Gadéa C., Pochic S., Cadres, classes moyennes : vers l'éclatement?, Paris, Armand Colin, pp. 116-126.

HatzFeld N. (2004), «L'individualisation des carrières à l'épreuve. Les grippages de la mobilité sur les chaînes de Peugeot-Sochaux», Sociétés contemporaines, Vol. 2, $n^{\circ} 54$, pp. 15-33.

Havard C. (2000), «La segmentation du système d'emploi : évolutions et enjeux pour la Poste», in Barreau J., Compeyron A., Havard C., Ménard J.-Y., Servel L., Une irrésistible modernisation des entreprises de service public?, Rennes, PUR, pp. 81-105.

JoIn-LAmbert O. (2001), Le receveur des postes, entre l'État et l'usager (1944-1973), Paris, Belin.

Join-LAmbert O. (2005), «Au nom de l'usager : les transformations du travail des receveurs des postes entre 1965 et 1974», Revue d'histoire moderne et contemporaine, no 52-4, pp. 183-204.

Lallement M. (2010), Le travail sous tensions, Paris, Éditions Sciences humaines.

Linhart D., Maruani M. (1982), «Précarisation et déstabilisation des emplois ouvriers », Travail et Emploi, no 11, pp. 27-36.

Mahouche B. (2007), «Gestion de la main-d'œuvre et insécurité professionnelle : l'exemple des employés du tri postal (1946-1980)», Interrogations, n 4, pp. 95-112.

Merllié D. (2006), «Comment confronter mobilité "subjective" et mobilité "objective"?», Sociologie $d u$ travail, Vol. 48, no 4, pp. 474-486.

Piotet F. (dir.) (2002), La révolution des métiers, Paris, PUF.

Strauss A. (1992), «La dynamique des professions», in La trame de la négociation. Sociologie qualitative et interactionniste, Paris, L'Harmattan.

Vezinat N. (2009), «Des contractuels davantage promus que les fonctionnaires », Personnel, no 503.

ZARIFIAN P. (2003), La cohabitation fonctionnaires et agents contractuels à la Poste. État des lieux et perspectives, Mission de la Recherche, Groupe La Poste. 